\title{
Alternating electric fields (TTFields) inhibit metastatic spread of solid tumors to the lungs
}

\author{
Eilon D. Kirson · Moshe Giladi · Zoya Gurvich · Aviran Itzhaki · \\ Daniel Mordechovich · Rosa S. Schneiderman · Yoram Wasserman • \\ Bernhard Ryffel · Dorit Goldsher · Yoram Palti
}

Received: 11 February 2009/ Accepted: 9 April 2009/Published online: 23 April 2009

(c) The Author(s) 2009. This article is published with open access at Springerlink.com

\begin{abstract}
Tumor treating fields (TTFields) are low intensity, intermediate frequency, alternating electric fields used to treat cancerous tumors. This novel treatment modality effectively inhibits the growth of solid tumors in vivo and has shown promise in pilot clinical trials in patients with advanced stage solid tumors. TTFields were tested for their potential to inhibit metastatic spread of solid tumors to the lungs in two animal models: (1) Mice injected with malignant melanoma cells (B16F10) into the tail vein, (2) New Zealand White rabbits implanted with VX-2 tumors within the kidney capsule. Mice and rabbits were treated using two-directional TTFields at 100 $200 \mathrm{kHz}$. Animals were either monitored for survival, or sacrificed for pathological and histological analysis of the lungs. The total number of lung surface metastases and the absolute weight of the lungs were both significantly lower in TTFields treated mice then in sham control mice. TTFields treated rabbits survived longer than sham control animals. This extension in survival was found to be due to an inhibition of metastatic spread, seeding or growth in the lungs of TTFields treated rabbits compared to controls. Histologically, extensive peri- and intra-tumoral immune
\end{abstract}

E. D. Kirson $(\varangle) \cdot$ M. Giladi · Z. Gurvich · A. Itzhaki ·

D. Mordechovich · R. S. Schneiderman · Y. Wasserman ·

Y. Palti

NovoCure Limited, Matam Advanced Technology Centre,

Topaz Bldg., 31905 Haifa, Israel

e-mail: Eilon@novo-cure.com

\section{B. Ryffel}

Laboratoire d'immunologie et Embryologie Mole' culaire, Rue de la Ferollerie, Centre National de la Recherche Scientifique, 45071 Orleans, France

D. Goldsher

Rambam Medical Center, P.O. Box 9602, 31096 Haifa, Israel cell infiltration was seen in TTFields treated rabbits only. These results raise the possibility that in addition to their proven inhibitory effect on the growth of solid tumors, TTFields may also have clinical benefit in the prevention of metastatic spread from primary tumors.

Keywords Tumor treating fields - Metastases . Immune response

\section{Abbreviation \\ TTFields Tumor treating fields}

\section{Introduction}

Pulmonary metastases, a common outcome of primary cancerous tumors, are present in $20-54 \%$ of all patients who die of cancer and are very often the cause of death of the patient $[1,2]$. The survival rate for patients with pulmonary metastases is dependent upon the location of the primary tumor, its histology and differentiation, number of lesions, the presence of mediastinal nodal disease and the resectability of the tumor [2-5]. The 3 years survival rate from diagnosis of pulmonary metastases is about $0 \%$ for patients with hepatocellular carcinoma [6] and the 3-year survival rate from diagnosis is $25 \%$ for patients with soft tissue sarcoma [3].

Examples of the prevalence of metastases include: epithelial (43\%), sarcoma (42\%), germ cell (7\%) and melanoma (6\%) [4]. The extrathoracic tumors that frequently metastasize to the lungs are: stomach, uterus, breast, colorectal, head-and-neck, and renal $[2,7,8]$.

Current treatments for pulmonary metastases include the following: metastasectomy, which is often the patient's 
best hope for cure, resulting in a 5 years survival rate of less than $50 \%$ in most cases [2]; chemotherapy and radiotherapy that are usually given as an adjunct to surgery with some beneficial effect but also hold a potential risk due to significant side effects [9]; hormonal therapy with a relatively low response rate [10]; thermal ablation which has shown promising results in preliminary experiments [9, $11,12]$ but holds the risk of excessive heat of the surrounding tissue; and finally, immuno-chemotherapy which has shown significant therapeutic efficacy, however, only in specific tumor types [13].

Despite all the progress made in past years, the clinical outcome of the above mentioned treatments is still poor. This could be attributed in part to tumor avoidance of immuno-editing [14]. The identification of tumor-associated antigens as 'self', leads to only a partial immune response and is one of the factors that limits the ability of the body to achieve recovery [15]. Furthermore, chemotherapy as well as stress induced by surgery are also factors that attenuate the immune system and weaken the immune response, thus further reducing the ability of the body to recover from these insults $[16,17]$.

Recently, a novel anti-cancer treatment modality, named Tumor Treating Fields (TTFields) was introduced. TTFields are low intensity, intermediate frequency alternating electric fields that were shown to disrupt cancer cell replication in vitro and to slow tumor progression in animal cancer models and patients with recurrent and newly diagnosed glioblastoma [18-20]. Yet, little is know about the effect of TTFields on the metastatic spread of solid tumors. The application of TTFields to animals and patients has been shown to have almost no acute or chronic toxicity $[18,20]$. Specifically, due to the relatively high frequency of TTFields $(>100 \mathrm{kHz})$, these fields do not stimulate nerves or muscles and thus have no effect on cardiac pacing. In addition, the low intensity used (single volts per $\mathrm{cm}$ ), does not cause significant heating within the body $[18,20]$. The purpose of the present work is to test the effect of TTFields on metastasis formation in two animal models and to investigate the development of an immune response following TTFields treatment.

\section{Materials and methods}

TTFields were tested for their potential to inhibit metastatic spread of solid tumors to the lungs in two animal models: malignant melanoma in mice and VX-2 tumors in rabbits. Animal experiments were conducted in accordance with the Technion-Israel Institute of Technology guidelines for the care of laboratory animals.

TTFields generating system as well as the electric fields measurement device were described elsewhere [18, 19].
The electric fields intensities are expressed in Volts (peak to peak) per $\mathrm{cm}$.

TTFields treatment of a melanoma lung metastases model in mice

Female C57BL/6 mice $(n=35)$, weighing $20 \pm 0.3 \mathrm{~g}$, were inoculated IV with a mouse malignant melanoma cell line (B16F10). Immediately following inoculation the mice were separated randomly into the two treatment groups. TTFields treatment was applied through four insulated electrodes placed around the mouse's torso (Fig. 1a). TTFields were applied in two perpendicular directions to the entire mouse torso with a duty cycle of $1: 1 \mathrm{~s}$. The TTFields frequency was set at $100 \mathrm{kHz}$, with currents of 90-100 mA which corresponds to electric field intensities of $1.8-2 \mathrm{~V} / \mathrm{cm}$ within the mouse abdomen and lungs. Control mice were treated by means of sham electrodes which were both temperature, and geometrically matched to the TTFields group. Treatment was applied for 7 days continuously and the mice were sacrificed for lung evaluation either 1 or 7 days after treatment termination. Metastatic load in the lungs was assessed by weighing the lungs and by counting the number of lesions seen on the external surface of the lungs (after replacing the blood with saline to improve visibility).
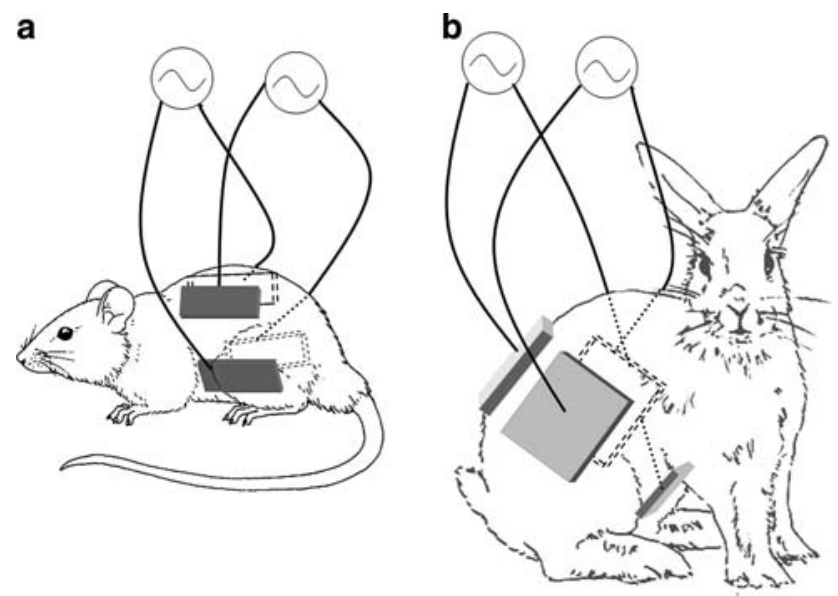

Fig. 1 Placement of electrodes on mice (a) and rabbits (b). The four insulated electrodes were attached to the skin using hydrogel after depilation. The electrodes were wrapped with leucoplast and electrodes wires were connected to the TTFields generating system or to the sham control system. The four electrodes were functionally divided into two pairs each generating one field direction through the animal. The electrode pairs were placed so as to create two perpendicular field directions at the center of body. Sham electrodes were placed in the same configuration 
Effect of TTFields therapy on the formation and growth of pulmonary metastases, and the local immune response in rabbits

Adult New Zealand White Rabbits weighting 2.5-3 kg were implanted with two fragments of VX-2 tumors of about $1 \times 1 \mathrm{~mm}$ each under the left kidney capsule of each rabbit under anesthesia. Two weeks after tumor implantation, baseline MRI was performed to detect the presence of tumor and to assess its baseline volume.

On the day following the baseline MRI, 4 electrode arrays each consisting of 9 insulated electrodes $\left(2.85 \mathrm{~mm}^{2}\right.$ each) connected in parallel were placed on torso of each tumor bearing animal of the treated group. The electrode arrays were placed on the abdomen, back and both flanks of the animal at the level of the kidneys (Fig. 1b). Identically shaped sham electrodes were placed on torso of each animal from the control group. Each sham electrode array was provided with an electrical heating element to match the temperature generated on the skin by the TTFields treatment electrodes. Both the treatment and the sham treatment electrodes included temperature sensors to monitor the temperature at the skin-electrode interface.

The animals in the treatment group were exposed to TTFields and those in the control group to heating. Six rabbits (in each experiment), were treated with TTFields for 5 weeks continuously. TTFields parameters were monitored and adjusted manually 1-3 times daily.

The rabbits were treated to the abdomen and retroperitoneum (but not lungs) using two directional $200 \mathrm{kHz}$ TTFields with average current of $790 \pm 80 \mathrm{~mA}$ (which correspond to $2.6 \pm 0.3 \mathrm{~V} / \mathrm{cm}$ in the kidney) and duty cycle of $1 \mathrm{~s}$. After each week of treatment electrodes were removed and all animals underwent T1 weighted MRI of the left kidney using a GI Genesis-Signa MRI device (at the Rambam Medical Center, Haifa, Israel). After the MRI the electrodes were replaced and treatment continued for a total treatment period of 35 days. Tumor volume assessment by MRI was performed by a technician blinded to the group of each rabbit.

Following treatment, animals were either followed for survival, or sacrificed for pathological and histological analysis of the primary kidney tumor and lung metastases.

\section{Data analysis}

Average tumor volumes in treated rabbits were compared to the average tumor volumes in the sham rabbits in each experiment. Statistical analysis of tumor growth rates, with reference to baseline was performed using the student $t$-test. The number of metastases and their size distribution were compared between groups using the Chi-squared test.
The survival time was taken as number of days from the implantation to the day the animal died. For both treated and control groups the median survival time was calculated. The statistical significance between the survival curves was analyzed using the Log-Rank test.

The subsequent parameters were inspected using microscopic investigation: tumor diameter, number of nodules per section and the extent of necrosis and infiltration by mononuclear cells. Histological slides from the lungs of TTFields treated and control rabbits were stained for immunological markers to demonstrate different populations of immune cells in the tissue. The extent of the intratumoral infiltration of the lymphocyte subsets was assessed by semi-quantitative score with increasing severity (0-5).

\section{Results}

TTFields treatment of a melanoma lung metastases model in mice

Continuous TTFields treatment for 7 days reduced the size and number of lung melanoma metastases at the end of treatment as compared to sham controls. Representative photos of lungs from treated and control mice are shown in Fig. 2a and b, respectively. The average number of surface metastases per lung in the TTFields group was significantly lower than in sham controls: $1.5 \pm 1$ compared to $10.3 \pm 8.4$, respectively (Fig. 2 c; $P<0.01$ ). The average lung weight of the TTFields treated animals was also significantly lower than the average lung weight in the control group (212 \pm 10 vs. $253 \pm 20 \mathrm{mg}$, respectively; $P<0.01$; see Fig. 2d).

When mice were followed for an additional week after termination of the TTField treatment, the overall number of lung metastases was similar in TTFields and sham control animals (18 \pm 12 vs. $17 \pm 15$, respectively; $P=0.87)$ and the lung weights were practically identical (370 \pm 28 vs. $365 \pm 40 \mathrm{mg}$, respectively; $P=0.79$ ). However, the distribution of metastasis sizes differed significantly between the groups $(P<0.05$, Chi-squared test $)$ with a higher proportion of large metastases seen in the control group, and a higher proportion of small metastases in the TTFields treated group (Fig. 3).

Effect of TTFields therapy on the formation and growth of pulmonary metastases, and the local immune response in rabbits

\section{Primary tumor}

The baseline average tumor volume in the sham control rabbits and in treated rabbits was identical (1.19 \pm 1.1 vs. 
Fig. 2 Malignant melanoma metastases as seen on the surface of the lungs of mice treated with TTFields. Exemplary photos of lungs of mice treated with TTFields (a) or sham control (b) are shown after removal of the pulmonary blood by perfusion with saline. Average number of surface metastases $( \pm \mathrm{SD})$ in treated and control mice (c). Average lung weight $( \pm \mathrm{SD})$ of treated and control mice a
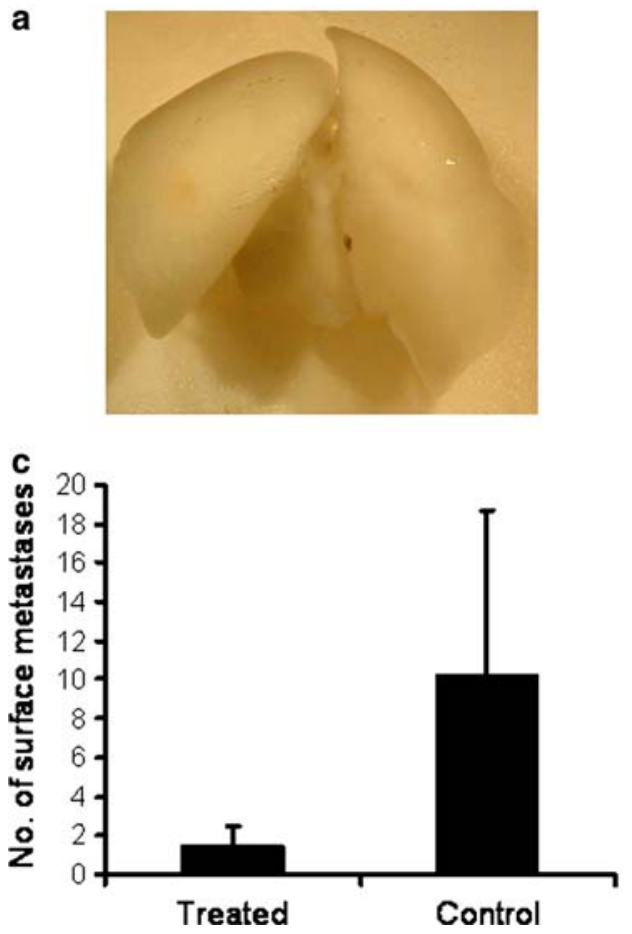

b
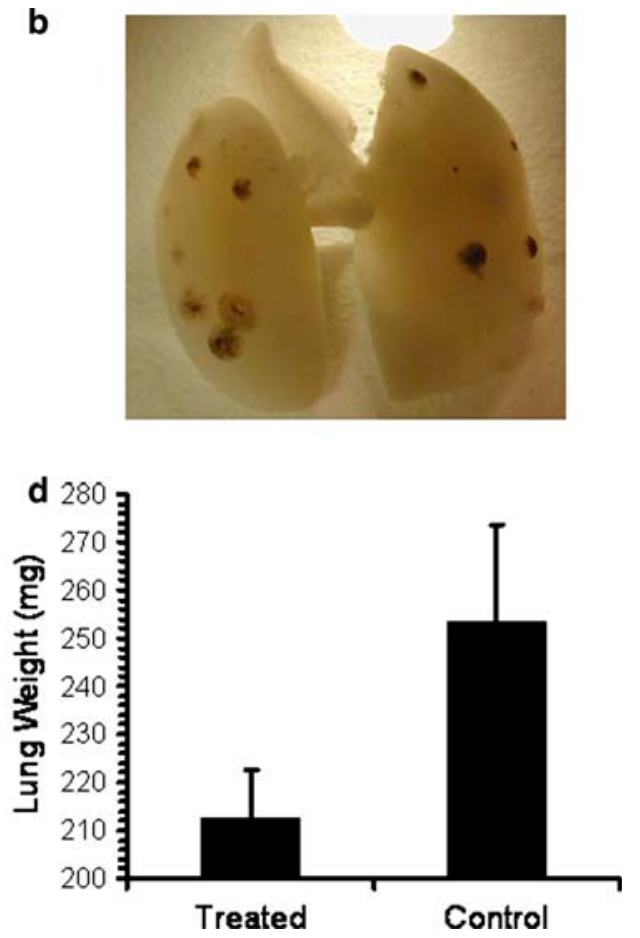

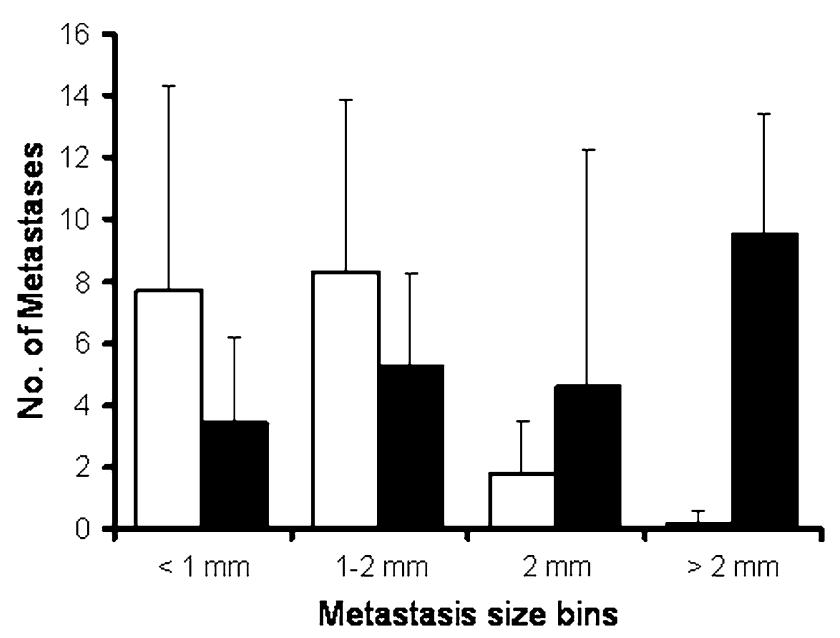

Fig. 3 Metastases number and size distribution in mice treated with TTFields (open bars) and sham control (closed bars). The mice were treated for 7 days followed by 7 days of recovery

$1.19 \pm 1.0 \mathrm{~cm}^{3}$, respectively; $\left.P=0.97\right)$. The average tumor volume after treatment was half of the volume of untreated sham controls $\left(29.0 \pm 18.5\right.$ vs. $61.1 \pm 38.5 \mathrm{~cm}^{3}$, respectively; $P=0.0011$ ). The average daily growth rate of the TTFields treated tumors was also significantly lower than that of the sham controls $(1.2 \pm 1.1$ vs. $3.5 \pm 3.2$, respectively; $P=0.003$ ). Figure 4 shows examples of T1 weighted MRI images (with gadolinium) of the maximal cross-sectional area of the tumor in representative treated versus sham control rabbits. The tumor in both images is hypo-intense with a ring-like contrast enhancement and necrotic center. The maximal tumor cross-section in the treated animal (Fig. 4a) is about half of that seen in the control animal (Fig. 4b).

\section{Animal survival}

The median survival of TTFields treated animals was 70 days which is significantly longer than the 57 days of the control animals (Log-Rank test; $P<0.05$ ). The Kaplan-Meier survival curve of the treatment results is shown in Fig. 5. This finding was surprising since the damage to the kidneys was unilateral in all animals. This fact together with the appearance of respiratory failure in most animals prior to their demise, led us to investigate the possibility of a difference in the metastatic load in TTFields treated versus sham control rabbits.

\section{Lung metastases}

The average TTFields intensities in the lung were $0.5 \mathrm{~V} / \mathrm{cm}$ which is a fifth of the field intensity measured in the kidney (see "Methods"). Despite the fact that the field intensity in the lungs was too low to have an inhibitory effect on tumors [19], when treatment was initiated before day 14 from implantation $(n=12)$, a significantly lower number of lung metastases was seen in TTFields treated rabbits than in sham controls (Fig. 6c; $P>0.05$ ). A trend towards a lower number of large metastases ( $>3 \mathrm{~mm}$ in diameter) was also seen in TTFields treated group, however, here the difference did not reach significance (Fig. $6 \mathrm{~d} ; P=0.11$ ). 
Fig. 4 T1 weighted MRI images (post gadolinium) of the maximal cross-sectional area of a TTFields treated (a) versus sham control tumor (b) in rabbit kidneys. Arrows indicate tumor location. Scale bar $1 \mathrm{~cm}$
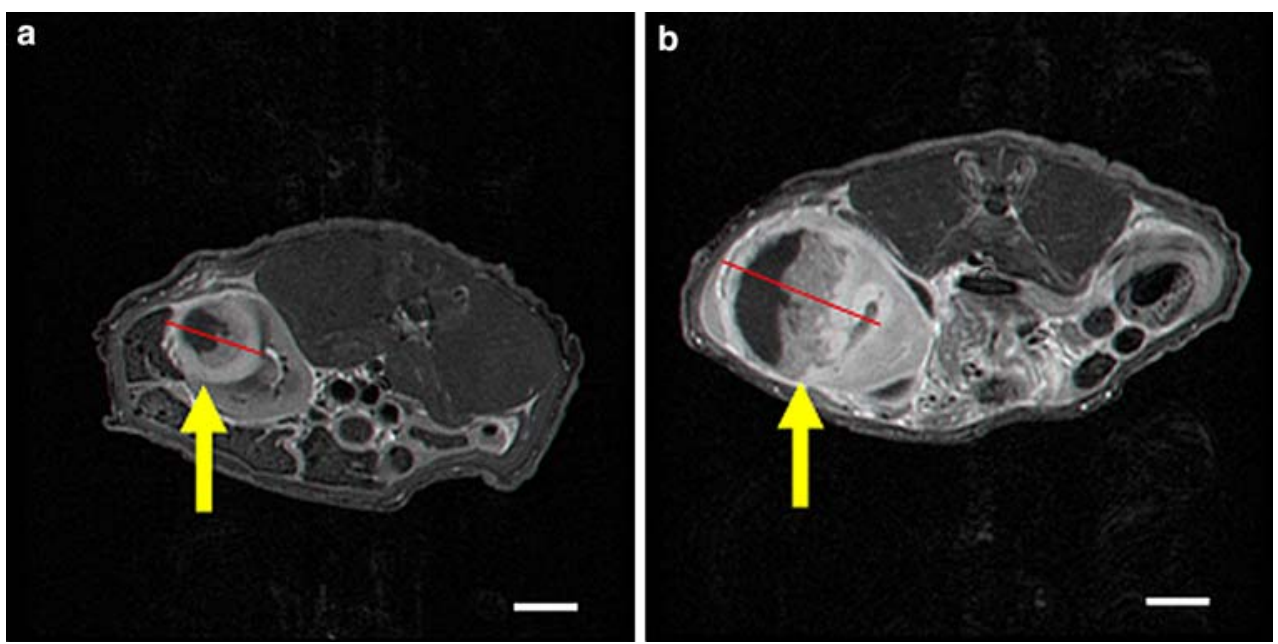

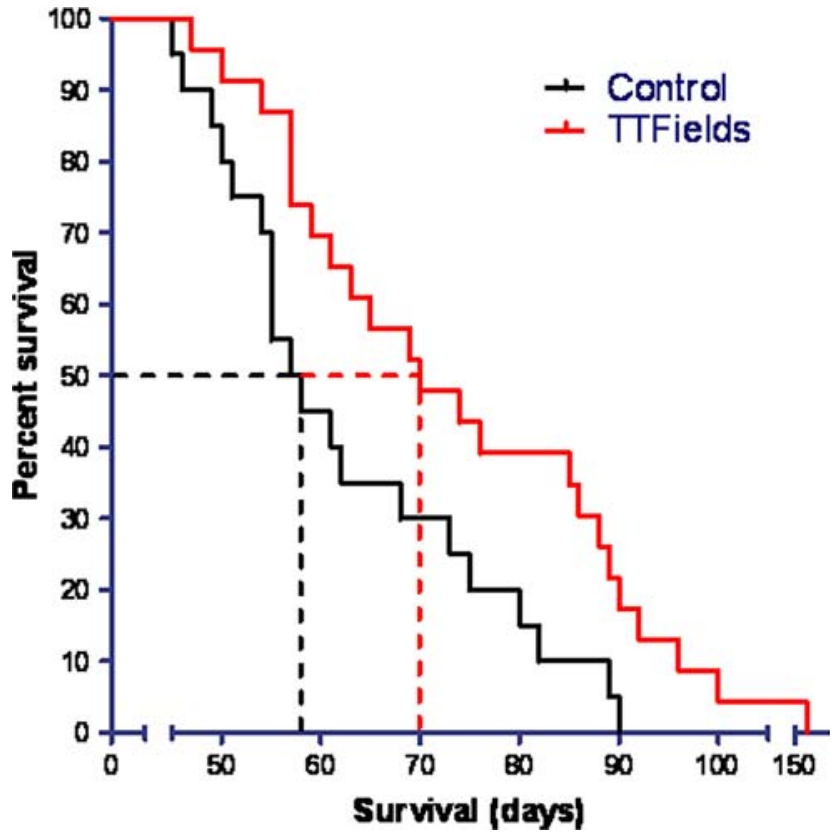

Fig. 5 Kaplan-Meier survival curve of rabbits treated with TTFields $(n=23$; red line $)$ versus sham controls $(n=20$; black line $)$. The median survivals of 70 vs. 57 days, respectively, are indicated by dotted lines

When treatment was started later than 14 days from implantation, no difference was seen in the number lung metastases between the groups.

Similar results were obtained by microscopic scanning of histological slides of lungs in TTFields treated versus sham control rabbits. The mean number of lung metastases per tumor cross-section was 11.8 in TTFields treated mice and 23.2 for the control group. Mean metastasis diameter was $1.4 \mathrm{~mm}$ per tumor cross-section in TTFields treated groups and $6.2 \mathrm{~mm}$ in the control group.

In the lung sections, it was noted in all preparations that a mononuclear cell infiltration was present around and within the metastases. The extent of this cell infiltration was more profound in TTFields treated rabbits compared to the controls with a mean score of 2.0 for the treatment group and 1.4 for the control group.

In order to better appreciate the local immune reaction within the region of the lung metastases, immuno-histochemical staining for lymphocyte subsets was performed. This investigation revealed that TTFields treated rabbits had significantly increased CD4, CD8, and CD45 T-cell counts (Table 1) as compared to control.

Interestingly, though most of the immune infiltration was seen in the peri-tumoral location, the most significant difference was the presence of an abundant intra-tumoral infiltration in TTFields treated rabbits. Representative immuno-histochemical staining of CD45 positive T-cells is shown in Fig. 7. Among the intra-tumoral infiltrating cells, CD4 T-cells were more prevalent than CD8 T-cells.

\section{Discussion}

Lung metastases are a common severe outcome of many primary tumors. Combined treatment consisting of pulmonary metastasectomy and chemotherapy is often the patient's best hope for cure, yet in most cases the 5 years survival rate is less than 50\% [2]. Thus, prevention of metastatic spread from primary tumors is of paramount importance.

Recently, a new treatment modality against cancerous cells was introduced-low intensity, intermediate frequency alternating electric fields or TTFields. These finely tuned electric fields are applied using a portable battery operated device (NovoTTF; NovoCure Ltd., Haifa, Israel) through insulated surface electrodes, and have been shown to inhibit the growth of primary solid tumors in both preclinical and clinical studies [18, 20]. 
Fig. 6 Exemplary photos of surface lung metastases in TTFields treated (a) versus sham control rabbits (b). Treatment was initiated on day 12 from implantation of the kidney tumor. The average total number $( \pm \mathrm{SD})$ of surface metastases (c) and the average number of large metastases $( \pm \mathrm{SD})(\mathbf{d})$ in control versus treated rabbits a

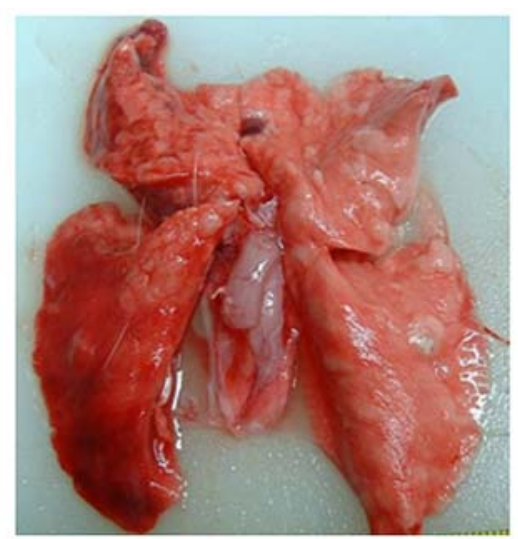

c

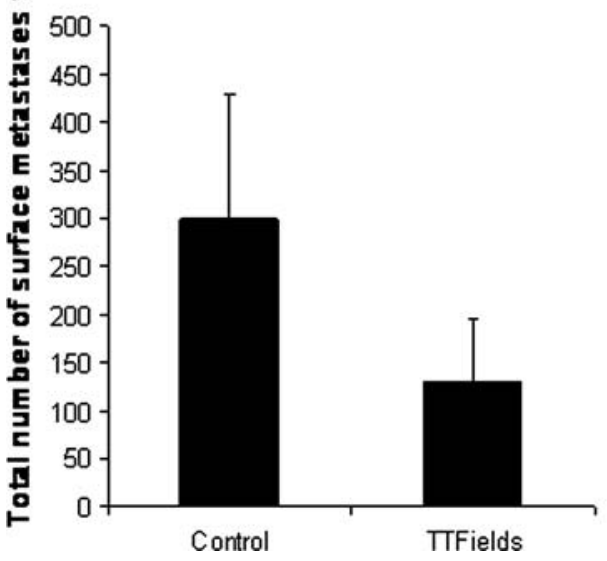

b

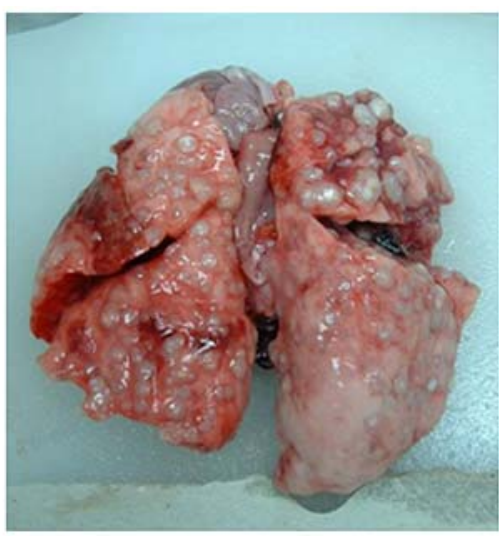

d

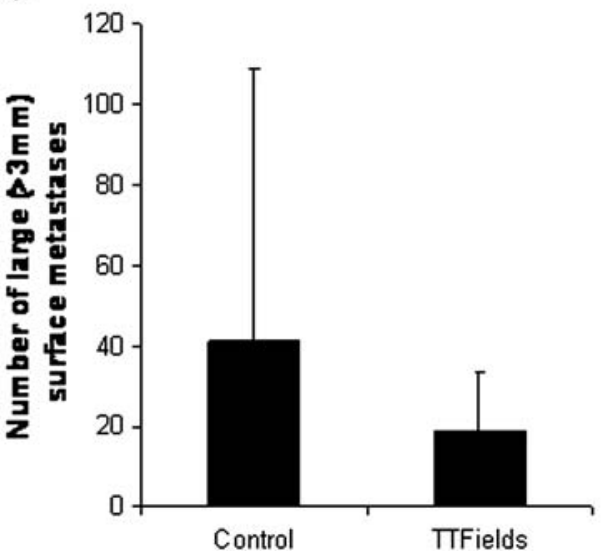

Table 1 Lymphocyte infiltration in lung tumors as revealed by immuno-histochemical staining

\begin{tabular}{lllll}
\hline Treatment & CD4 & CD8 & CD45 & CD19 \\
\hline Control & $2.0 \pm 0.8$ & $0.8 \pm 0.5$ & $2.0 \pm 0.8$ & 0 \\
TTFields & $3.4 \pm 0.9$ & $1.6 \pm 0.5$ & $3.6 \pm 0.5$ & 0 \\
\hline
\end{tabular}

\section{Metastases in mice}

In a preliminary attempt to study the effect of TTFields on metastatic lesions we tested the fields' effect when applied directly to the lung shortly after B16F10 melanoma cells were injected into the tail vein. After 7 days of TTFields application, the number of surface lung metastases in the TTFields treated mice was significantly reduced, compared to the sham control group. This result could be interpreted in several ways: the TTFields could have eliminated the B16F10 melanoma cells shortly after injection, or merely prevented their implantation in the lung. Alternatively, the implantation of the B16F10 melanoma cells in the lungs was not affected but the tumor progression was inhibited by the TTFields. The latter option is supported by the fact that 1 week after stopping treatment the number of lung metastases in the TTFields group was equal to the number observed in the sham control group. Furthermore, the different size distribution between the two groups suggests that the metastases progression was inhibited in the TTFields group during treatment, an effect which may have been attenuated or partially lost after 1 week recovery.

Inhibition of VX-2 carcinoma in rabbits' kidneys

Previously we reported that TTFields treatment significantly reduced progression of malignant melanoma and adenocarcinoma tumors in mice as well as glioma cells inoculated intracranially in Fischer rats [18, 19]. In the present study we demonstrate that TTFields can inhibit the growth of VX-2 carcinoma in the kidneys of New Zealand white rabbits. The MRI results illustrate that TTFields application significantly reduced tumor growth rate throughout treatment.

Though the inhibition of renal tumor growth was significant, it was not expected to result in such a noteworthy difference in the overall survival between the treatment group and the control. An alternative explanation was sought, that could account for the observed differences in the overall survival. Hence, we investigated the metastatic spread of the VX-2 tumors to the lungs [21]. 


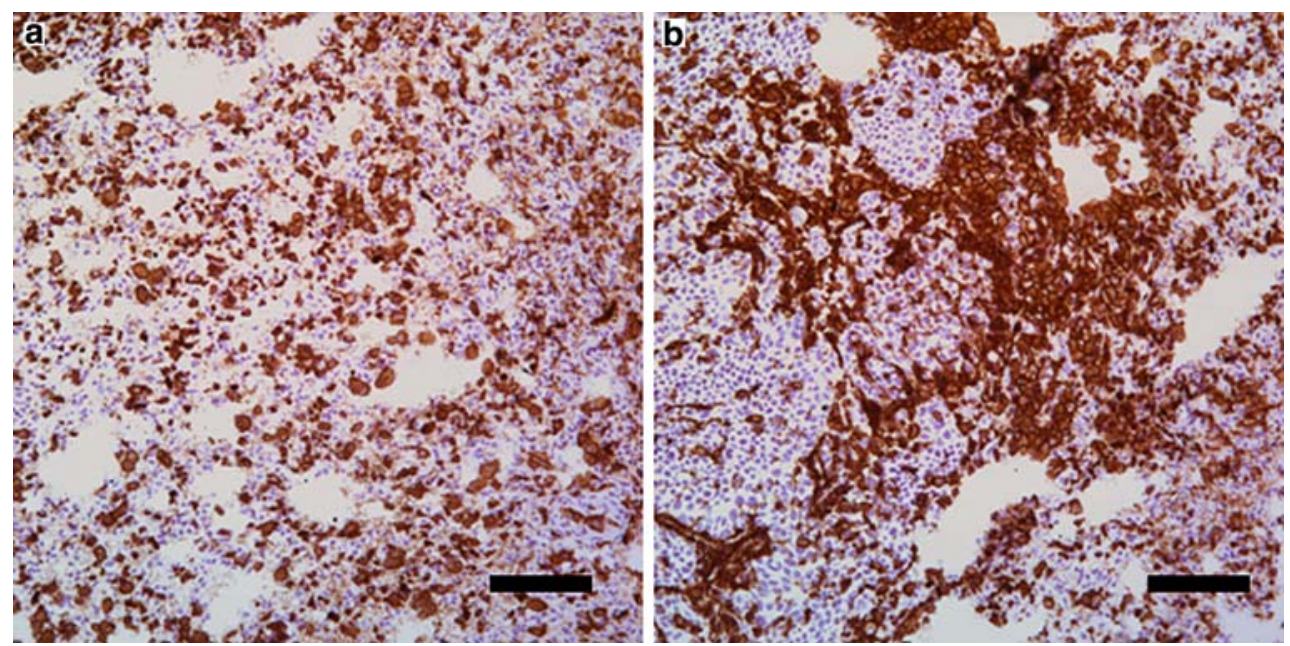

Fig. 7 Discrete intra-tumoral infiltration of CD45 positive T cells in control tumours (a) and abundant intra-tumoral CD45 positive T cells in TTFields treated tumours (b). Scale bar $100 \mu \mathrm{m}$

Metastases in rabbits

The TTFields intensity in the lungs was about $20 \%$ of the fields' intensity in the kidney (data not shown). Such a $0.5 \mathrm{~V} / \mathrm{cm}$ field intensity measured in the rabbit' lungs is below the threshold required for cancerous cell growth inhibition [19]. Since the TTFields application could not account for the decrease in the number of metastases, we searched for an alternative explanation for the results.

One such explanation relates to the inhibition of growth of the primary renal tumor. It is possible that by lowering the tumor load in the kidney, the metastatic potential of the tumor would decrease proportionately. Another possibility is based on the reported finding that there is an increase in metastatic spread of VX-2 tumors from the kidney between days 12 and 15 from implantation [21]. Here we report that treating the primary tumor with TTFields after day 15 from implantation resulted in a smaller difference between treated and control rabbit in the number of lung metastases. Therefore, it is possible that the inhibition of metastatic spread is due to an inhibitory effect on the capability of the tumor cells to migrate into the circulation of the primary tumor. Such migration is dependant on the proper formation of a microtubule based processes in the migrating cells [22]. Although we did not test this directly, the known inhibition of microtubule polymerization by TTFields during mitosis may have a similar effect on cancer cell migration and endothelial penetration. Future experiments are warranted to test this point directly.

An additional explanation for our findings is the enhancement of a systemic immune response to the tumor cells. This option is supported by the results of the immunostaining assay performed on treated and sham control lungs. It is becoming widely accepted that immune response can inhibit the proliferation of cancerous cell or even eliminate them (reviewed by Dunn et al. [23]), and thus account for the reduction in the number of metastases. Naturally, the following question would be: How could TTFields activate the immune response in an organ distant from the location where the fields are applied? Zitvogel et al. [14] reviewed several mechanisms through which conventional treatments could modulate the interaction between the tumor and the immune system: reduction of the tumor mass as a result of chemotherapy, surgery or radiation could reduce the tumor immunosuppressive properties; tumor cell destruction induced by chemotherapy or radiation could expose hidden tumor antigens; stimulation of the immune system through activation of immune effectors and regulatory mechanisms or by inducing lymphopenia followed by proliferation of immune effectors. Taken together, a self generated vaccination against cancer specific antigens may develop-leading to metastasis destruction by the immune system [14]. In addition, we found that the cells infiltrating the lung metastases in the TTFields group were CD4, CD8 and CD45 positive. This milieu of immune markers indicates a T-cell mediated immune response; however, it is not yet sufficient to prove a tumor-antigen specific immune reaction. Interestingly, CD45 activation can induce the production of $\mathrm{TNF} \alpha$ [24] which in turn activates a family of cell-surface receptors that can mediate cell death.

Assuming that the activation of the immune response in the rabbits' lungs was mediated by the TTFields treatment, a question arises regarding the TTFields effect on the immune system in the mice. The observation that the metastases renewed their growth in the lungs, once the TTFields application to the mice was terminated, does not support immune response activation in the treated group. It 
seems more likely that the inhibition of the metastases in the mice lungs was due to a direct inhibitory effect of the TTFields on the cancerous cells. Unlike the rabbits in which there was a substantial difference between the fields' intensities in the lungs and the kidneys, in the mice lungs the TTFields intensity was sufficiently large to induce inhibition of the metastasis. Why wasn't the immune system activated in the mice? The shorter treatment duration (1 week in mice compared to 5 weeks in rabbits) might not suffice for the induction of the immune response in mice. Differences between the cancerous cell lines used (VX-2 carcinoma in rabbits and B16F10 melanoma in mice) as well as differences between the animals' immune system could also account for these results. In addition, the tumors subjected to TTFields application in the rabbits were established large tumors which provided a larger target for the immune system and possibly presenting more tumor specific antigens after the TTFields treatment.

In conclusion, we have shown that TTFields have the potential to inhibit the migration of metastases from a primary tumor, can inhibit the growth of metastases in the lungs once they have been seeded in the target organ, by the presence of the fields in the lungs themselves, and finally, TTFields may activate an anti-tumor antigen systemic immune response following treatment of a primary tumor. Therefore, TTFields may not only be clinically useful in treatment of locally advanced tumors, but in prevention and treatment of metastatic disease as well.

Open Access This article is distributed under the terms of the Creative Commons Attribution Noncommercial License which permits any noncommercial use, distribution, and reproduction in any medium, provided the original author(s) and source are credited.

\section{References}

1. Friedel G (2007) Pulmonary metastasectomy: specific surgical considerations and prognostic factors. In: Schil P (ed) Lung metastases and isolated lung perfusion. Nova Science Publishers Inc., New York, pp 45-60

2. Sternberg DI, Sonett JR (2007) Surgical therapy of lung metastases. Semin Oncol 34(3):186-196. doi:10.1053/j.seminoncol. 2007.03.004

3. Billingsley KG, Burt ME, Jara E et al (1999) Pulmonary metastases from soft tissue sarcoma: analysis of patterns of diseases and postmetastasis survival. Ann Surg 229(5):602-610. doi: 10.1097/00000658-199905000-00002 discussion 10-2

4. Friedel G, Pastorino U, Buyse M et al (1999) Resection of lung metastases: long-term results and prognostic analysis based on 5,206 cases - the International registry of lung metastases. Zentralbl Chir 124(2):96-103

5. Khan JH, McElhinney DB, Rahman SB et al (1998) Pulmonary metastases of endocrine origin: the role of surgery. Chest 114(2):526-534. doi:10.1378/chest.114.2.526

6. Zhang S, Zeng Z, Tang Z et al (2008) Prognostic analysis of pulmonary metastases from hepatocellular carcinoma. Hepatol Intl 2(2):237-243
7. Pastorino U, Buyse M, Friedel J et al (1997) Long-term results of lung metastasectomy: prognostic analyses based on 5,206 cases. The International registry of lung metastases. J Thorac Cardiovasc Surg 113(1):37-49. doi:10.1016/S0022-5223(97)70397-0

8. Sardari N, Colpaert C (2007) Lung metastases: epidemiology, clinical presentation and imaging. In: Schil P (ed) Lung metastases and isolated lung perfusion. Nova Science Publishers Inc., New York, pp 3-25

9. Rose SC, Thistlethwaite PA, Sewell PE et al (2006) Lung cancer and radiofrequency ablation. J Vasc Interv Radiol 17(6):927-951 quiz 51

10. Lavrenkov K, Man S, Geffen DB et al (2002) Experience of hormonal therapy with anastrozole for previously treated metastatic breast cancer. Isr Med Assoc J 4(3):176-177

11. Suh R, Reckamp K, Zeidler M et al (2005) Radiofrequency ablation in lung cancer: promising results in safety and efficacy. Oncology 19(11, Suppl 4):12-21 Williston Park

12. VanSonnenberg E, Shankar S, Morrison PR et al (2005) Radiofrequency ablation of thoracic lesions: part 2, initial clinical experience-technical and multidisciplinary considerations in 30 patients. AJR Am J Roentgenol 184(2):381-390

13. Atzpodien J, Kirchner H, Illiger HJ et al (2001) IL-2 in combination with IFN- alpha and 5-FU versus tamoxifen in metastatic renal cell carcinoma: long-term results of a controlled randomized clinical trial. Br J Cancer 85(8):1130-1136. doi:10.1054/bjoc. 2001.2076

14. Zitvogel L, Apetoh L, Ghiringhelli F et al (2008) The anticancer immune response: indispensable for therapeutic success? J Clin Invest 118(6): 1991-2001. doi:10.1172/JCI35180

15. Whiteside TL (2008) The tumor microenvironment and its role in promoting tumor growth. Oncogene 27(45):5904-5912. doi: 10.1038/onc. 2008.271

16. McMillan TJ, Hart IR (1987) Can cancer chemotherapy enhance the malignant behaviour of tumours? Cancer Metastasis Rev 6(4):503-519. doi:10.1007/BF00047465

17. Walton B (1979) Effects of anaesthesia and surgery on immune status. Br J Anaesth 51(1):37-43. doi:10.1093/bja/51.1.37

18. Kirson ED, Dbaly V, Tovarys F et al (2007) Alternating electric fields arrest cell proliferation in animal tumor models and human brain tumors. Proc Natl Acad Sci USA 104(24):10152-10157. doi:10.1073/pnas.0702916104

19. Kirson ED, Gurvich Z, Schneiderman R et al (2004) Disruption of cancer cell replication by alternating electric fields. Cancer Res 64(9):3288-3295. doi:10.1158/0008-5472.CAN-04-0083

20. Kirson ED, Schneiderman RS, Dbaly V et al (2009) Chemotherapeutic treatment efficacy and sensitivity are increased by adjuvant alternating electric fields (TTFields). BMC Med Phys 9(1):1. doi:10.1186/1756-6649-9-1

21. Nakada SY, Jerde TJ, Warner T et al (2004) Comparison of cryotherapy and nephrectomy in treating implanted VX-2 carcinoma in rabbit kidneys. BJU Int 94(4):632-636. doi:10.1111/j. 1464-410X.2004.05015.x

22. Suresh S (2007) Biomechanics and biophysics of cancer cells. Acta Biomater 3(4):413-438. doi:10.1016/j.actbio.2007.04.002

23. Dunn GP, Bruce AT, Ikeda $\mathrm{H}$ et al (2002) Cancer immunoediting: from immunosurveillance to tumor escape. Nat Immunol 3(11):991-998. doi:10.1038/ni1102-991

24. Hayes AL, Smith C, Foxwell BM et al (1999) CD45-induced tumor necrosis factor alpha production in monocytes is phosphatidylinositol 3-kinase-dependent and nuclear factor-kappaBindependent. J Biol Chem 274(47):33455-33461. doi:10.1074/ jbc.274.47.33455 